\title{
ECOLOGICAL
} MODELLING

\section{Emergy evaluation of three cropping systems in southwestern Australia}

\author{
Edward Lefroy $^{\mathrm{a}, *}$, Torbjörn Rydberg ${ }^{\mathrm{b}}$ \\ ${ }^{a}$ CSIRO Sustainable Ecosystems, P.O. Box 5, Wembley, WA 6913, Australia \\ b Department of Ecology and Crop Production Science, Swedish University of Agricultural Sciences, Box 7047, S-750 07 Uppsala, Sweden
}

Received 19 July 2001 ; received in revised form 29 July 2002; accepted 19 September 2002

\begin{abstract}
Wind erosion and rising water tables are serious threats to the ecological sustainability of annual plant-based farming systems on deep, infertile sandplain soils in southwestern Australia. In this study, an annual cropping system was compared with two novel perennial plant-based systems designed to address these threats in terms of their use of renewable indigenous resource, their use of non-renewable indigenous resources, their purchased inputs of energy and materials, and profitability. The farming systems were an annual lupin/wheat (Lupinus angustifolius L./Triticum aestivum L.) crop rotation, a plantation of the fodder tree tagasaste (Chamaecytisus proliferus $\mathrm{L}$.) and an alley cropping system in which the lupin/wheat rotation was grown between spaced rows of tagasaste trees. Flows of energy and materials between the environment and the economy were identified for each farming system and the natural and human activity involved in generating inputs as goods or services then valued in terms of the equivalent amount of solar energy required for their production using the emergy method of Odum [Ecological and General Systems: An Introduction to Systems Ecology. University Press of Colorado, revised edition of Systems Ecology, 1983, Wiley, New York, 644 pp.; Environmental Accounting: Emergy and Environmental Decision Making. Wiley, New York, 370 pp.]. The results showed that the two largest energy flows in the conventional lupin/wheat cropping system were wind erosion and purchased inputs of phosphate. The renewable component of production was 15\% of total flows in the lupin/wheat system, $30 \%$ in the alley cropping system and $53 \%$ in the tagasaste plantation. The annual net income from the plantation system was nearly four times higher, and from alley cropping $45 \%$ higher, than from the lupin/wheat rotation. This analysis suggested that once the two agroforestry systems were fully established, the tagasaste plantation was the most efficient at transforming natural resources into goods and services and the most profitable, while the lupin/wheat system was the least energy efficient and the least profitable.
\end{abstract}

(c) 2002 Elsevier Science B.V. All rights reserved.

Keywords: Energy analysis; Emergy; Wind erosion; Water use: Tagasaste; Chamaecytisus proliferus; Southwestern Australia

\section{Introduction}

The development of agriculture in southwestern Australia since European settlement 170 years ago

\footnotetext{
${ }^{*}$ Corresponding author. Tel.: +61-8-9333-6442; fax: $+61-8-9333-6444$.

E-mail address: ted.lefroy@cse.csiro.au (E. Lefroy).
}

has involved the rapid replacement of the endemic woodland, heath and forest vegetation with a synthetic annual grassland of crops and pastures. The sustainability of these farming systems is now in question in light of their environmental impact and this has prompted interest in the development of better adapted farming systems (Williams, 1997; Lefroy et al., 2000). 
Two consequences of vegetation change have been wind erosion and rising water tables. The semi-arid climate, low relief and sandy surfaced soils have meant that large wind erosion events have become a feature of crop growing areas since clearing. Rising water tables are a direct consequence of the lower annual transpiration of agricultural crops compared to the original woodland and heath vegetation. Lower transpiration in turn produces higher rates of runoff and deep drainage and results in water logging, and on some heavier textured soils with high levels of salt storage in the subsoils, land salinisation (Hatton and Nulsen, 1999). Water logging and salinity currently affect $10 \%$ of the 20 million ha of cleared land in southwestern Australia and this is predicted to increase $30 \%$ by the middle of next century (Hatton and Nulsen, 1999). This is affecting not only the productivity of farmland but also water quality, built infrastructure and the viability of remnant stands of native vegetation (George et al., 1997). In the area of our study, a 2 million ha sandplain region lying between latitudes 28 and $31^{\circ}$ south on the west coast of Australia, the major water related issue is water logging rather than salinity.

Emphasis has therefore been placed on surface soil protection and increased water use in the development of alternative farming systems, and there is increasing recognition that meeting these objectives will involve a significant increase in the proportion of deep-rooted perennial plants in the agricultural landscape (Hatton and Nulsen, 1999). A major obstacle to the development and adoption of perennial-based land use systems such as forestry and agroforestry is the difficulty of quantifying and demonstrating their relative economic and ecological sustainability. Their commercial and environmental impacts are long term and therefore less easily predicted. Their environmental impacts vary with their stage of maturity and are less open to modification once in place. They also represent more expensive mistakes than the adoption of innovations in annual farming systems if they prove to be unacceptable for economic, ecological, or other reasons.

An important question is therefore how to identify land use systems that have the best likelihood of long term success. Wind erosion control, water management and profitability alone are insufficient criteria for sustainability. While it is widely recognized that assessment of sustainability requires integrat- ing ecological, economic and social factors, decision making is currently based on a fragmented process that lacks a common language. A general definition of sustainable agriculture is "the ability to maintain production over long time frames despite major ecological and socio-economic perturbations and stress" (Conway, 1985; Altieri, 1987). Economic sustainability is commonly assessed by calculating profitability, cash flow and returns on investment. Resources and resource degradation are also occasionally valued directly in economic terms although placing a dollar value on ecosystem services and their disruption remains problematic (Pearce, 1983; Pearce and Turner, 1990; Costanza et al., 1997; Pimentel et al., 1997; Edwards-Jones et al., 2000). Ecological sustainability can be assessed by comparing carbon, nitrogen and water cycling and impacts on biodiversity at species, community and landscape scales. The experience of rapid land degradation in southwestern Australia highlights the need for more integrated approaches to quantifying the impact of farming systems on the environment and the human economy. In this study, the emergy method of energy accounting developed by Odum $(1994,1996)$ was selected as it offers a means of quantifying the direct and indirect environmental work involved in generating a product or service. The aims of this study were to compare the resource use and environmental impact of two novel perennial plant-based farming systems with those of conventional annual cropping with a particular emphasis on wind erosion control and water use.

\section{Method}

\subsection{Emergy analysis}

The use of energy analysis to value transactions between human society and nature was initially based on the work of Lotka (1925) and gained prominence in the 1970s, but has suffered from two main failings. First, while conventional energy analysis accounts for the direct and indirect fuel consumption needed to produce a good or service, it provides no information about its potential impact or appropriate use (Fluck and Baird, 1980; Stout, 1990; Smil, 1991). Secondly the language of energy is not easily translated into the currency of human exchange. 
Emergy analysis was developed as a form of environmental accounting to address these weaknesses. Odum $(1994,1996)$ defined as a measure of the total energy used in the past to make a product or service, as distinct from the contemporary available energy expressed by the heat content in Joules. This goes beyond classical energetics in recognizing different qualities or forms of energy, acknowledging that energy of one dilute form (such as sunlight) is used to generate more concentrated forms, such as plant products. In doing so, it makes allowance for the fact that different kinds of energy, such as a calorie of sunlight, electricity or human thinking, represent different abilities to do work.

By attempting to account for all the work previously involved in generating a resource, product or service, the emergy method sets out to provide a scientific basis for wealth. The common basis for evaluating all flows and storages of energy and materials is solar emergy, defined as the amount of available solar energy used up directly and indirectly to make a service or product, expressed as salar emJoules (sej). A fundamental organizing principle of the emergy concept is the maximum power principle, which is stated according to Odum (1996) "In the competition among self-organizing processes, network designs that maximize power will prevail." Brown and Herendeen (1996) stated the maximum power principle as follows: "Systems that will prevail in competition with others develop the most useful work from in-flowing emergy sources by reinforcing productive processes and overcoming limitations through system organization." As a consequence, it is suggested that the convergence of energy through the network of transformations necessary to generate a product or service results in that product or service being supported by more emergy than is reflected in its available energy.

The ratio of emergy required to make a product or service to the available energy of the product or service is defined as the transformity. The units of transformity are solar emJoules/Joule, abbreviated sej/J or solar emJoules $/ \mathrm{kg}$ (sej/kg). The higher the transformity, the higher that item is located in the energy hierarchy chain. This is based on the assumption implicit in the maximum power principle that the more energy required to make a product or service, the higher its emergy value. The emergy of a product or service is calculated by multiplying its available energy by its transformity. For some commodities, different transformities have been derived in different contexts. The transformity calculated under the most similar conditions to those in a particular study should be chosen in order to arrive at the best estimate of the emergy value for a product or service. Where no transformity exists from a previous study for an essential component, a new transformity has to be derived. In this study for example an emergy analysis of the Australian national economy was carried out to derive a transformity for the Australian dollar.

A potential advantage of this method over conventional economic and energy analysis is the capacity to value renewable and non-renewable natural resource inputs and environmental service outputs normally considered 'free'. A fuller treatment of the theoretical background to the emergy approach can be found in Odum (1998), Brown and Ulgiati (1998), Odum et al. (2000), Brown et al. (2000), Brown and Ulgiati (2001) and Odum and Odum (2001).

\subsection{The farming systems}

The systems under study were a lupin/wheat rotation, an alley cropping system in which this crop rotation is grown between rows of the fodder tree tagasaste (Chamaecytisus proliferus Link) $30 \mathrm{~m}$ apart (550 trees/ha) and plantation density tagasaste (2300 trees/ha). The lupin/wheat rotation represents the conventional cropping system in the study area, a region characterized by deep, infertile sandy soils on the south west coast of Australia. The plantation system has been adopted on an estimated 100000 ha in this region over the last decade for cattle and sheep production (Tudor, personal communication). The alley cropping system is a more recent innovation, developed by landholders as a multi-purpose system to protect their core grain growing activity by reducing wind erosion and deep drainage while simultaneously providing supplementary stock feed in autumn (Lefroy, 1994). In this study, it was assumed that trees in alley and plantation systems were cut and grazed in autumn, in accordance with commercial practice, and that income from the tree fodder was derived by agisting stock at a carrying capacity of one dry sheep equivalent (DSE) per kilogram of edible tagasaste dry matter per day (Oldham et al., 1994). In the 
lupin/wheat and alley cropping systems, the grazing value of crop residues was also included.

\subsection{Conceptual approach}

Analysis was carried out at a 1 ha scale for each of the three farming systems. The flows of matter and energy across each system boundary are shown in aggregate form in Fig. 1 using the energy systems symbols described in Odum $(1994,1996)$. Seen from this perspective, agriculture is driven by two kinds of outside sources. To the left are the free renewable emergy flows (sun, wind and rain), and to the right the purchased sources of emergy in the form of fuel, goods and services. These are shown aggregated into renewable environmental inputs $(R)$, non-renewable environmental inputs $(N)$, fuel and materials $(M)$, the service component of purchased inputs $(S)$ and finally the total emergy $(Y)$ being the energy required to support the yield. These aggregated flows of emergy were then used to derive indices of sustainability and returns on invested emergy as a basis for comparing the alternative land uses (Fig. 1). The indices used are renewable portion of total emergy $(R / Y)$; environmental loading $(N+M+S) / R$; investment ratio $(M+S) /(R+N)$; emergy exchange ratio $Y /$ (output price in emergy); return on invested emergy (output price in emergy) $/(M+S)$.

The solar emergy of each flow was calculated by multiplying the energy in Joules (or directly from its mass) by specific transformities which attempt to account for the amount of solar emergy required to make

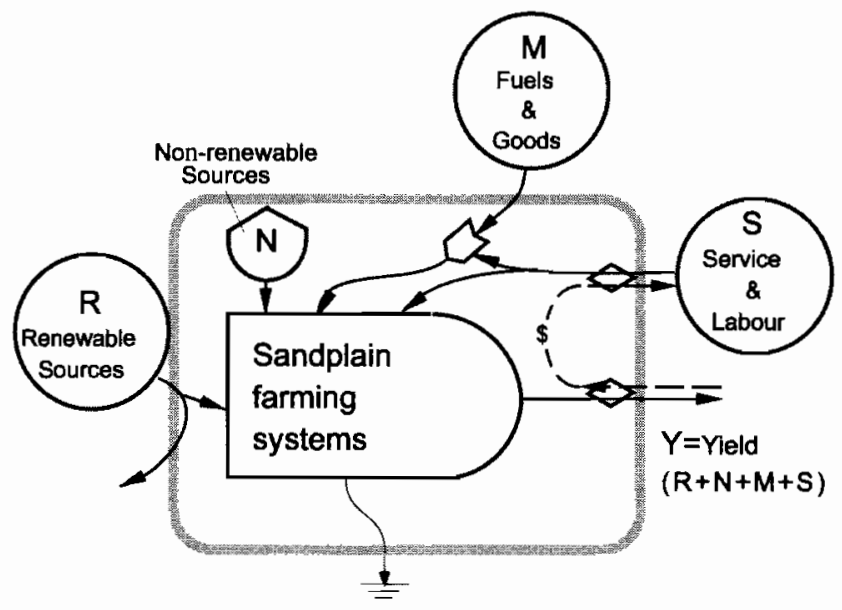

SUSTAINABILITY INDICATORS

$\begin{array}{ll}\text { Renewable proportion of total emergy used } & R / \\ \text { Ratio of renewable to non-renewable emergy } & (N+M) / R \\ \text { Environmental loading } & (N+M+S) / R\end{array}$

RETURN ON INVESTED EMERGY Investment ratio

Emergy exchange ratio

$(M+S) /(R+N)$

Return on investment emergy

$\mathrm{Y} /$ (output price in emergy)

(output price in emergy)/(M+S)

Fig. 1. Systems diagram illustrating the flow of energy and materials to and from a sandplain farming system expressed as flows of solar emergy per unit time where emergy is the cumulative measure of the energy used in the past to make a product or service, expressed in units of solar energy (solar emJoules; sej), (Odum, 1996). The aggregated flows shown are local renewable inputs $(R)$, non-renewable storages $(N)$, purchased energy and materials $(M)$ and the service component of purchased inputs $(S)$ and $(Y)$ which is the sum of the emergy value of the inputs. The aggregated flows are used to calculate emergy-based indices of sustainability. 
a product or service, expressed as solar emJoules per Joule (sej/J) or solar emJoules per gram ( $\mathrm{sej} / \mathrm{g}$ ). These transformities are derived from previous studies that have evaluated the energy flows and conversion efficiencies involved in producing the natural resource, product or service inputs (Odum, 1996). According to this definition, the transformity should express the solar energy per unit available energy (Odum, 1996). The new transformities generated in this study are expressed as solar emergy per Joule, with the measure of energy being based on the enthalpy value for the new calculated transformities with the exception of nitrogen which is expressed on a weight basis (sej/kg).

\subsection{Data sources and emergy evaluation}

The data were taken primarily from an 8 ha farming systems experiment comparing productivity, water use and nitrogen inputs at a site $200 \mathrm{~km}$ north east of Perth, Western Australia (Lefroy and Stirzaker, 1999; Lefroy et al., 2001a,b; Unkovich et al., 2000). Assumptions about the management and productivity of each farming system and data sources used in the analyses are listed in footnotes to Tables 1-5.

To place an emergy value on the service component of purchased inputs, we calculated the emergy to dollar ratio for the Australian economy in the 1996/1997 financial year. This was done by dividing the total emergy value of Australia's Gross National Product (GNP) by its dollar value. The emergy value of Australia's GNP was calculated from commodity statistics for Australia's products and exports for the year 1996 (Australian Bureau of Agricultural and Resource Economics, 1997) and transformities reported by Odum (1996). The emergy to money ratio for Australia 1996/1997 was found to be $2.71 \times$ $10^{12} \mathrm{sej} / \mathrm{US} \$$ (calculations available from the authors).

\section{Results}

\subsection{Emergy analysis of the farming systems}

The emergy flows calculated for the three land use systems are itemized in Tables 1-5 and represented in aggregated form in Fig. 2. Emergy analysis of the lupin/wheat rotation system (Tables 1 and 2) shows that largest emergy flows were associated with soil
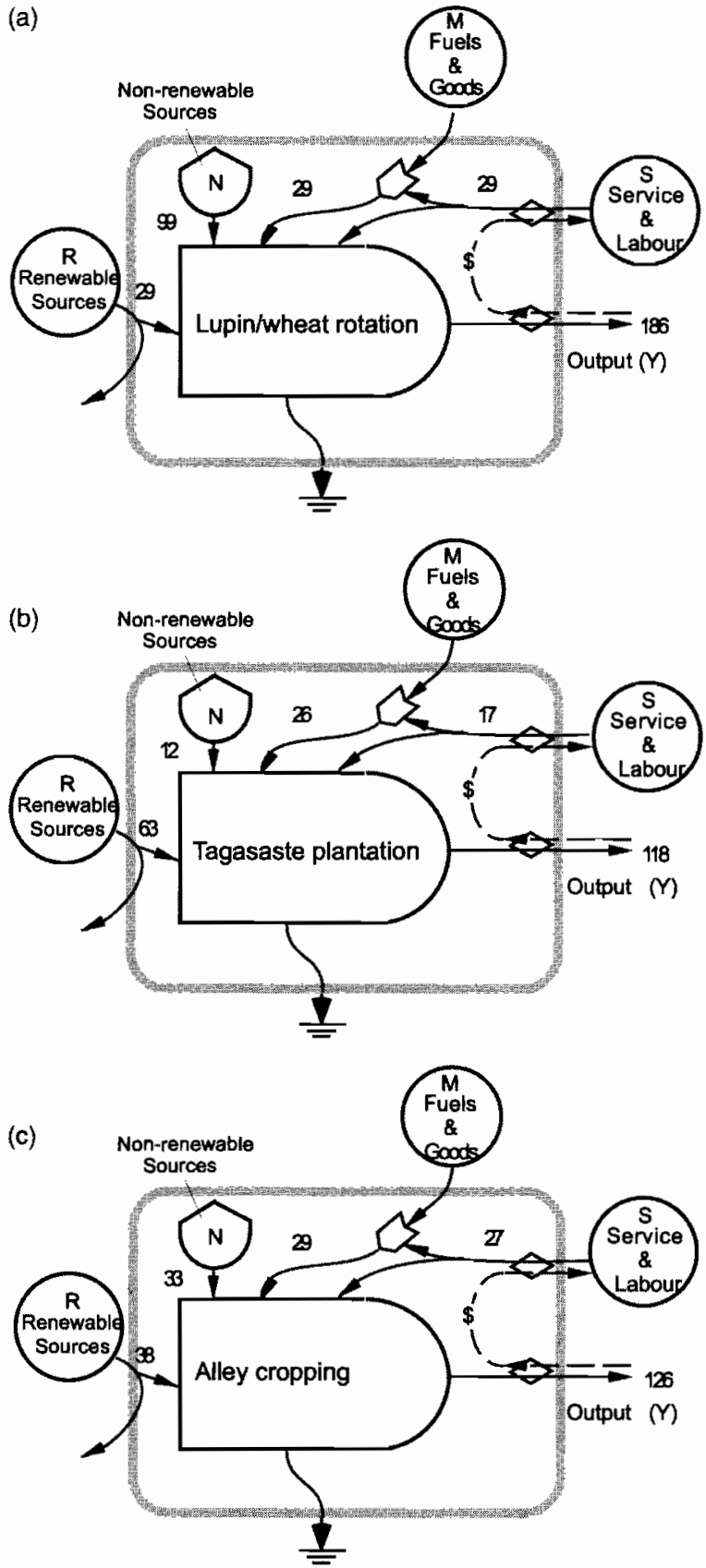

Fig. 2. Summary diagram of the emergy flows in the three cropping systems: (a) lupin/wheat rotation, (b) tagasaste plantation and (c) alley cropping. All emergy flows are $10^{13} \mathrm{sej} / \mathrm{ha}$ per year. The largest of the renewable energy sources $(R$, items $1-4$, Tables 1-5), is taken to represent total renewable flows as the other renewable energy flows represent by-products of the same coupled processes. 
Table 1

Emergy evaluation of lupin/wheat rotation (all flows per hectare per year)

\begin{tabular}{|c|c|c|c|c|}
\hline ltem number & Item description & $\begin{array}{l}\text { Annual flow } \\
\text { (raw units) }\end{array}$ & $\begin{array}{l}\text { Transformity } \\
\text { (sej/unit) }\end{array}$ & $\begin{array}{l}\text { Solar emergy } \\
\left(\text { sej } \times 10^{12}\right)\end{array}$ \\
\hline \multicolumn{5}{|c|}{ Renewable energy sources $(R)$} \\
\hline 1 & Sunlight $(J)$ & $5.66 \mathrm{E}+13$ & $1.0 \mathrm{E}+00$ & 56.6 \\
\hline 2 & Wind, kinetic energy $(\mathrm{J})$ & $2.72 \mathrm{E}+10$ & $1.5 \mathrm{E}+-03$ & 40.7 \\
\hline 3 & Rain, chemical energy $(\mathbf{J})$ & $1.58 \mathrm{E}+10$ & $18.2 \mathrm{E}+03$ & 287.7 \\
\hline 4 & Rain, geopotential energy $(\mathrm{J})$ & $9.02 \mathrm{E}+08$ & $10.5 \mathrm{E}+03$ & 9.5 \\
\hline \multicolumn{5}{|c|}{ Non-renewable sources (internal) $(N)$} \\
\hline 5 & Net topsoil loss (J) & $1.58 \mathrm{E}+10$ & $62.5 \mathrm{E}+03$ & 987.5 \\
\hline \multicolumn{5}{|c|}{ Purchased fuels and goods $(M)$} \\
\hline 6 & Diesel, gasoline and lubricants ( $\mathbf{J})$ & $3.05 \mathrm{E}+08$ & $56.1 \mathrm{E}+03$ & 17.1 \\
\hline 7 & Electricity (J) & $3.60 \mathrm{E}+06$ & $173.7 \mathrm{E}+03$ & 0.6 \\
\hline 8 & Phosphate (kg) & $1.29 \mathrm{E}+01$ & $17.0 \mathrm{E}+12$ & 219.1 \\
\hline 9 & Pesticides (kg) & $2.72 \mathrm{E}+08$ & $56.1 \mathrm{E}+03$ & 15.3 \\
\hline 10 & Seed $(J)$ & $1.10 \mathrm{E}+09$ & $34.8 \mathrm{E}+03$ & 38.3 \\
\hline 11 & Machinery and buildings (kg) & $1.17 \mathrm{E}+00$ & $3.0 \mathrm{E}+12$ & 3.5 \\
\hline \multicolumn{5}{|c|}{ Labor and service $(S)$} \\
\hline 12 & Labor (US\$) & $1.34 \mathrm{E}+01$ & $2.4 \mathrm{E}+12$ & 32.3 \\
\hline 13 & Diesel, gasoline and lubricants (US\$) & $2.98 \mathrm{E}+00$ & $2.4 \mathrm{E}+12$ & 7.2 \\
\hline 14 & Electricity (USS) & $5.00 \mathrm{E}-01$ & $2.4 \mathrm{E}+12$ & 1.2 \\
\hline 15 & Phosphate (US\$) & $2.10 \mathrm{E}+01$ & $2.4 \mathrm{E}+12$ & 50.6 \\
\hline 16 & Pesticides (US\$) & $1.59 \mathrm{E}+01$ & $2.4 \mathrm{E}+12$ & 38.3 \\
\hline 17 & Seed (US\$) & $2.97 \mathrm{E}+01$ & $2.4 \mathrm{E}+12$ & 71.6 \\
\hline 18 & Machinery and buildings (US\$) & $2.79 \mathrm{E}+01$ & $2.4 \mathrm{E}+12$ & 67.3 \\
\hline \multirow[t]{2}{*}{19} & Consultants, insurance and interest (US\$) & $9.40 \mathrm{E}+00$ & $2.4 \mathrm{E}+12$ & 22.7 \\
\hline & Total service and labor (US\$) & $1.21 \mathrm{E}+02$ & & 291.1 \\
\hline \multicolumn{5}{|l|}{ Output $(Y)$} \\
\hline 20 & Grain $(\mathrm{J})$ & $1.59 \mathrm{E}+10$ & $117.0 \mathrm{E}+03^{\mathrm{a}}$ & 1860.3 \\
\hline 21 & Crop residues $(\mathrm{J})$ & $1.29 \mathrm{E}+10$ & $144.2 \mathrm{E}+03^{\mathrm{b}}$ & 1860.3 \\
\hline 22 & Nitrogen fixation $(\mathrm{kg})$ & $4.00 \mathrm{E}+01$ & $46.5 \mathrm{E}+12^{\mathrm{c}}$ & 1860.3 \\
\hline \multicolumn{5}{|c|}{ Economic value of output } \\
\hline 23 & Grain (US\$) & $1.48 \mathrm{E}+02$ & $2.4 \mathrm{E}+12$ & 355.2 \\
\hline \multirow[t]{2}{*}{24} & Crop residues (US\$) & $2.24 \mathrm{E}+01$ & $2.4 \mathrm{E}+12$ & 53.8 \\
\hline & Total value (US\$) & $1.70 \mathrm{E}+02$ & & \\
\hline
\end{tabular}

See Table 2 for numbers and its description.

a Solar transformity of grain (sej/J).

b Solar transformity of crop residues (sej/J).

c Solar transformity of nitrogen fixation $(\mathrm{sej} / \mathrm{kg})$.

erosion (item 5), evapotranspiration (item 3) and phosphate fertilizer (item 8). Those three sources accounted for more than $80 \%$ of the total emergy budget. Emergy in labor and service (items 12-19) accounted for 16\% of the total emergy budget. In the tagasaste plantation system (Tables 3 and 4), emergy in evapotranspiration (item 3 ) was the single largest driving force followed by phosphorus (item 8 ) and soil loss (item 5). In alley cropping, evapotranspiration, soil loss and phosphorus
(Table 5) were also the three largest emergy sources, although soil loss was reduced to one-third of that in the lupin/wheat system due to the presence of the spaced tree rows. Labor, pesticides, seed, diesel, machinery and buildings were, in emergy terms, almost 1 order of magnitude lower than the largest sources in all the farming systems.

Soil erosion, the highest emergy flow in the lupin/wheat system, had an annual flow of $988 \mathrm{E}+$ 
Table 2

\begin{tabular}{ll}
\hline Item number & ltem description \\
\hline 1 & Average insolation at Moora $7.25 \times 10^{9} \mathrm{~J} / \mathrm{m}^{2}$ per year (Lefroy and Stirzaker, 1999). Energy received over \\
& land $=10000 \mathrm{~m}^{2}$ (land area) $\times 7.25 \times 10^{9} \mathrm{~J} / \mathrm{m}^{2}$ per year $\times(1-0.22)\left(1-\right.$ albedo annual crop) $=5.66 \times 10^{13} \mathrm{~J}$ \\
& per year. Transformity $=1$ by definition (Odum, 1996). \\
& Eddy diffusion coefficient assumed to be $2.8 \mathrm{~m}^{3} / \mathrm{m} / \mathrm{s}$ (Odum and Odum, 1983); vertical velocity gradient estimated \\
& from 18 locations around Australia, $5000 \mathrm{~m} / \mathrm{m} / \mathrm{s}$ (Australian Bureau of Meteorology, 1977). Wind energy \\
& absorbed $=10000 \mathrm{~m}^{2} \times 1000 \mathrm{~m}\left(\right.$ height of boundary layer) $\times 1.23 \mathrm{~kg} / \mathrm{m}^{3}$ (density of air) $\times 2.8 \mathrm{~m}^{3} / \mathrm{m} / \mathrm{s} \times$ \\
& $3.154 \times 10^{7} \mathrm{~s}$ per year $\times\left(5 \times 10^{-3}\right) \mathrm{m}^{2} / \mathrm{m} / \mathrm{s}=2.72 \times 10^{10} \mathrm{~J}$ per year. Transformity from Odum $(1996)$. \\
& Precipitation $=460 \mathrm{~mm}$ per year. evapotranspiration of annual crops $=320 \mathrm{~mm}$ per year $($ Asseng et al., 1998; \\
& Lefroy and Stirzaker, 1999). Chemical potential energy of rain over land = $10000 \mathrm{~m}^{2}$ (land area) $\times 0.32 \mathrm{~m}$ \\
& (evapotranspiration) $\times 1000 \mathrm{~kg} / \mathrm{m}^{3}$ (density of water) $\times 4940 \mathrm{~J} / \mathrm{kg}$ (Gibbs free energy of rainwater) $=$ \\
& $1.5808 \times 10^{10} \mathrm{~J}$ per year. Transformity from Odum (1996).
\end{tabular}

Runoff assumed to be $\cong 1 \%$ of precipitation on these deep, coarse sands $=4.6 \mathrm{~mm}$ per year. Geopotential energy of rain $=0.046 \mathrm{~m}$ (runoff) $\times 10000 \mathrm{~m}^{2}$ (land area) $\times 200 \mathrm{~m}$ (mean elevation of land mass) $\times 1000 \mathrm{~kg} / \mathrm{m}^{3}$ (density of water) $\times 9.8 \mathrm{~m} / \mathrm{s}^{2}$ (gravity) $=9.016 \times 10^{8} \mathrm{~J}$ per year. Transformity from Odum (1996).

Average soil loss from wind erosion assumed to be $1 \mathrm{~mm} / \mathrm{ha}$ per year (being a $5 \mathrm{~mm}$ loss from a major erosion event on average every 5 years) $=14$ tha per year at a bulk density of $1.4,5 \%$ of which is organic matter (including $10-100 \mathrm{~kg} / \mathrm{ha}$ nitrogen), based on research by Carter et al. (1992) and Carter (unpublished data). Energy of soil loss $=10000 \mathrm{~m}^{2}$ (farmed area) $\times 1.4 \mathrm{~kg} / \mathrm{m}^{2}$ per year (erosion rate) $\times 0.05 \%$ (organic matter) $\times$ $5400 \mathrm{kcal} / \mathrm{kg} \times 4186 \mathrm{~J} / \mathrm{kcal}=1.58 \times 10^{10} \mathrm{~J} / \mathrm{ha}$ per year. Transformity from Odum (1996). Diesel consumption $=11 \mathrm{l} / \mathrm{h}$ for machinery operation. Operating hours per hectare of crop $=(2 \times$ spraying $\times 20 \mathrm{ha} / \mathrm{h}+$ $1 \times$ seeding $\times 8 \mathrm{ha} / \mathrm{h}+1 \times$ harvest $\times 6 \mathrm{ha} / \mathrm{h})=23.5 \mathrm{~min} / \mathrm{ha} .1 \mathrm{l} \mathrm{l} / \mathrm{h} \times 23.5 / 60=4.31 \mathrm{l} / \mathrm{ha}$ per year. Energy content $=4.31 \mathrm{l} / \mathrm{ha} \times 5.05 \times 10^{7} \mathrm{~J} / 1=2.18 \times 10^{8} \mathrm{~J} / \mathrm{ha}$ year. Gasoline consumption involved in running $2000 \mathrm{ha}$ farm $=30001$ per year $=1.5 \mathrm{l} / \mathrm{ha}$ year. Energy content $=1.5 \mathrm{l} / \mathrm{ha}$ per year $\times 5.42 \times 10^{7} \mathrm{~J} / 1=8.13 \times 10^{7} \mathrm{~J} / \mathrm{ha}$ per year. Annual consumption of lubricants $=201 / 100 \mathrm{~h}$ machinery time $\times 23.5 \mathrm{~min} / \mathrm{ha}$ per year $=0.078 \mathrm{l} / \mathrm{ha}$ per year. Energy content $=0.0781 /$ ha per year $\times 7.38 \times 10^{7}=5.76 \times 10^{6} \mathrm{~J} /$ ha per year. Total energy use $=3.05 \times 10^{8} \mathrm{~J} / \mathrm{ha}$ per year. Transformities from Odum (1996). Annual consumption involved in running $2000 \mathrm{ha}$ farm $=2000 \mathrm{kWh}=1.0 \mathrm{kWh} / \mathrm{ha}$ per year. Energy content $=1.0 \mathrm{kWh} / \mathrm{ha}$ per year $\times 3.6 \times 10^{6} \mathrm{~J} / \mathrm{kWh}=3.6 \times 10^{6} \mathrm{~J} / \mathrm{ha}$ per year. Transformity from Odum (1996). Annual application $150 \mathrm{~kg} / \mathrm{ha} \times 8.6 \%, \mathrm{P}=12.9 \mathrm{~kg} / \mathrm{ha}$ per year. Transformity from Odum (1996) service subtracted. Herbicides $2.0 \mathrm{~kg} / \mathrm{ha}$ per year $\left(2.5 \mathrm{~kg} / \mathrm{ha}\right.$ lupins, $1.5 \mathrm{~kg} / \mathrm{ha}$ cereals) $\times 9.1 \times 10^{7} \mathrm{~J} / \mathrm{kg}=1.82 \times 10^{8} \mathrm{~J} / \mathrm{ha}$ per year (oil equivalent) $+1.70 \mathrm{~kg} / \mathrm{ha}$ per year $\times 5.30 \times 10^{7} \mathrm{~J} / \mathrm{kg}=9.01 \times 10^{7} \mathrm{~J} / \mathrm{ha}$ per year, total $=2.721 \times 10^{8} \mathrm{~J} / \mathrm{ha}$ per year. Transformity for refined oil products from Odum (1996). Lupins $100 \mathrm{~kg} / \mathrm{ha}$, oats $75 \mathrm{~kg} / \mathrm{ha}$. Mean seeding rate $87.5 \mathrm{~kg} / \mathrm{ha}$ per year. Energy content $=3000 \mathrm{kcal} / \mathrm{kg} \times$ $87.5 \times 4186 \mathrm{~J} / \mathrm{kcal}=1.10 \times 10^{9} \mathrm{~J} / \mathrm{ha}$ per year. Transformity for industrial corn production, service subtracted (Odum and Odum, 1983).

Total weight of machinery used in crop production $=2 \mathrm{t}($ tractor $)+3 \mathrm{t}$ (header) $+1 \mathrm{t}$ (seeder) $+0.55 \mathrm{t}($ boom spray) $=$ $6550 \mathrm{~kg}$. Assume a life of 7 years and $1000 \mathrm{ha} \mathrm{crop}=0.94 \mathrm{~kg} / \mathrm{ha}$ per year. Buildings-machinery shed

$(18 \mathrm{~m} \times 9 \mathrm{~m})$, super shed $(18 \mathrm{~m} \times 9 \mathrm{~m}), 3 \times 150 \mathrm{kl}$ silos, total weight $7 \mathrm{t}$ steel for a 1000 ha crop program in any one year $=7.00 \mathrm{~kg} / \mathrm{ha}$ per year. Assume a life of 30 years $=0.23 \mathrm{~kg} / \mathrm{ha}$ per year. Transformity for refined steel products, service subtracted (Odum and Odum, 1983). Average farm income after costs and tax in 1996/1997 US\$20000 (mean for cropping areas, ABARE, 1997) $=$ US $\$ 10 \mathrm{ha}^{-1}$ crop. Contract labor for cropping program $=0.4 \mathrm{~h} / \mathrm{ha} \times \mathrm{US} \$ 8.40 \mathrm{~h}^{-1}=\mathrm{US} \$ 3.36 \mathrm{ha}^{-1}$. Total costs US\$ $13.36 \mathrm{ha}^{-1}$. Transfornity used is the emergy/money ratio for the Australian economy 1996/1997 (Rydberg and Lefroy, unpublished data).

Diesel consumption $=11 \mathrm{l} / \mathrm{h}$ for machinery operation. Operating hours per hectare of crop $=$ $(2 \times$ spraying $\times 20 \mathrm{ha} / \mathrm{h}+1 \times$ seeding $\times 8 \mathrm{ha} / \mathrm{h}+1 \times$ harvest $\times 6 \mathrm{ha} / \mathrm{h})=23.5 \mathrm{~min} / \mathrm{ha} .11 \mathrm{l} / \mathrm{h} \times 23.5 / 60=4.31 \mathrm{l} / \mathrm{ha}$ per year. Cost $=4.31 \mathrm{l} / \mathrm{ha} \times$ US $\$ 0.46 \mathrm{I}^{-1}=\mathrm{US} \$ 2.00 \mathrm{ha}^{-1}$ per year. Gasoline consumption involved in running

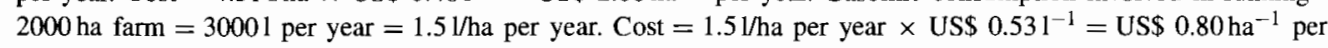
year. Annual consumption of lubricants $=20 \mathrm{~V} 100 \mathrm{~h}$ machinery time $\times 23.5 \mathrm{~min} / \mathrm{ha}$ per year $=0.0781 / \mathrm{ha}$ per year. Cost $=0.0781 /$ ha per year $\times$ US $\$ 2.50 \mathrm{l}^{-1}=$ US $\$ 0.175 \mathrm{ha}^{-1}$ per year. Transformity see item 12 above. Annual consumption involved in running 2000 ha farm $=2000 \mathrm{kWh}=1.0 \mathrm{kWh} / \mathrm{ha}$ per year. Cost $=1.0 \mathrm{kWh} / \mathrm{ha} \mathrm{per}$ year $\times$ US $\$ 0.5 / \mathrm{kWh}=$ US $\$ 0.5 \mathrm{ha}^{-1}$ per year. Transformity see item 12 above. Annual application $150 \mathrm{~kg} / \mathrm{ha} \times 8.6 \%, \mathrm{P}=12.9 \mathrm{~kg} / \mathrm{ha}$ per year. Cost US $\$ 140 \mathrm{t}^{-1}=\mathrm{US} \$$ $0.14 \mathrm{~kg}^{-1} \times 150 \mathrm{~kg} / \mathrm{ha}=$ US $\$ 21 \mathrm{ha}^{-1}$ per year. Transformity see item 12 above. 
Table 2 (Continued)

\begin{tabular}{|c|c|}
\hline Item number & Item description \\
\hline 16 & $\begin{array}{l}\text { Herbicides } 2 \mathrm{~kg} / \mathrm{ha} \text { per year } \times \text { US } \$ 7 \mathrm{~kg}^{-1}=\mathrm{US} \$ 14 \mathrm{ha}^{-1} \text { per year (oil equivalent) }+ \text { insecticides } 0.17 \mathrm{~kg} / \mathrm{ha} \text { per } \\
\text { year } \times \text { US } \$ 11.20 \mathrm{~kg}^{-1}=\text { US } \$ 1.90 \mathrm{ha}^{-1} \text { per year, total }=\text { US } \$ 15.90 \mathrm{ha}^{-1} \text { per year. Transformity see item } 12 \text { above. }\end{array}$ \\
\hline 17 & $\begin{array}{l}\text { Cos } 1 \text { of seed; lupins } 100 \mathrm{~kg} / \mathrm{ha} \text {, US } \$ 0.28 \mathrm{~kg}^{-1}=\mathrm{US} \$ 28 \mathrm{ha}^{-1} \text { every second year. Oats } 75 \mathrm{~kg} / \mathrm{ha} \text {, US } \$ \\
0.42 \mathrm{~kg}^{-1}=\text { US } \$ 31.50 \mathrm{ha}^{-1} \text { every second year. Mean seeding cost }=\text { US } \$ 29.70 \mathrm{ha}^{-1} \text { per year. Transformity see } \\
\text { item } 12 \text { above. }\end{array}$ \\
\hline 18 & $\begin{array}{l}\text { Total annual cost of owning and maintaining machinery used in crop production }=\text { annual depreciation }+ \text { interest } \\
\text { on half the amount owing on a loan if it is paid off over the life of the machinery }+ \text { interest on the salvage value }+ \\
\text { maintenance costs. Assume current value in US to be US } \$ 28000 \text { (tractor) }+ \text { US } \$ 17500 \text { (seeder) }+ \text { US } \$ 70000 \\
\text { (header) }+ \text { US } \$ 10500 \text { (boom spray) }=\text { US } \$ 126000 \text {. Assume this has a life of } 7 \text { years, a salvage value at the end } \\
\text { of that time of US } \$ 35000 \text {, interest rate on borrowing are } 15 \% \text {, which adjusted for inflation of } 5 \text { is } 10 \% \text {. } \\
\text { Depreciation }=(126000-35000) / 7=\text { US } \$ 13000 \text {. Interest }=(126000-35000) / 2 \times 10 \%=\text { US } \$ 4550 \text {. Interest } \\
\text { on salvage value }=\text { US } \$ 35000 \times 10 \%=\text { US } \$ 3500 \text {. Assume maintenance costs }=\text { US } \$ 4000 \text {. Total costs }=\text { US } \$ \\
13000+4550+3500+5000=\text { US } \$ 26550 \text {. Assume } 1000 \text { ha crop }=\text { US } \$ 26.55 \text { ha }^{-1} \text { per year. Annual cost of } \\
\text { buildings = replacement cost/life }=\text { US } \$ 40000 / 30=\text { US } \$ 1300=\text { US } \$ 1.30 \text { ha }^{-1} \text { per year). Transformity see item } \\
12 \text { above. }\end{array}$ \\
\hline 19 & $\begin{array}{l}\text { Farm management consultant paid US } \$ 1400 \text { per year }=\text { US } \$ 1.40 \mathrm{ha}^{-1} \text { per year. Insurance }=\text { US } \$ 1000 \text { per } \\
\text { year = US } \$ 0.50 \mathrm{ha}^{-1} \text { per year. Average farm debt in the cropping zone is US\$150000 per year. With interest rate } \\
\text { adjusted for inflation annual cost }=\text { US } \$ 15000 \text { per year }=\text { US } \$ 7.50 \mathrm{ha}^{-1} \text { per year. Transformity see item } 12 \text { above, }\end{array}$ \\
\hline 20 & $\begin{array}{l}\text { Lupin yield } 800 \mathrm{~kg} / \mathrm{ha} \times 1.39 \times 10^{7} \mathrm{~J} / \mathrm{kg}=1.11 \times 10^{10} \mathrm{~J} / \mathrm{ha} \text {. Cereal yield } 1500 \mathrm{~kg} / \mathrm{ha} \times 1.39 \times 10^{7} \mathrm{~J} / \mathrm{kg}=2.08 \times 10^{10} \mathrm{~J} / \mathrm{ha} \text {. } \\
\text { Mean annul }=\left(3.19 \times 10^{10} \mathrm{~J} / \text { ha per year }\right) / 2=1.59 \times 10^{10} \mathrm{~J} / \text { ha per year. }\end{array}$ \\
\hline 21 & $\begin{array}{l}\text { Energy value of mean biomass production (straw and stubble) }=4000 \mathrm{~kg} / \mathrm{ha} \times 0.03 \mathrm{kcal} / \mathrm{kg} \times 4186 \mathrm{~J} / \mathrm{kg}=1.21 \times \\
10^{10} \mathrm{~J} / \mathrm{ha} \text { per year-most of which is indigestible. Unharvested grain }=0.05 \times 1.59 \times 10^{10} \mathrm{~J} / \mathrm{ha} \text { per year }=0.08 \times \\
10^{10} \mathrm{~J} / \mathrm{ha} \text { per year. Total }=1.29 \times 10^{10} \mathrm{~J} / \mathrm{ha} \text { per year. }\end{array}$ \\
\hline 22 & Assumes $80 \mathrm{~kg} / \mathrm{ha}$ fixed in the lupin phase every second year, average $40 \mathrm{~kg} \mathrm{~N} / \mathrm{ha}$ per year (Anderson et al., 1998). \\
\hline 23 & $\begin{array}{l}\text { Calculation based on US\$ } 142 \mathrm{t}^{-1} \text { for lupins and US\$ } 121 \mathrm{t}^{-1} \text { for wheat with yields of } 0.8 \text { and } 1.5 \mathrm{t} / \mathrm{ha} \text { respectively. } \\
\text { Mean income per year over the rotation is US\$ } 147.55 \text { per year. Transformity see item } 12 \text { above. }\end{array}$ \\
\hline 24 & $\begin{array}{l}\text { Grazing value is in the unharvested grain. Based on a maintenance requirement of } 3.6 \times 10^{6} \mathrm{~J} \text { per day, unharvested } \\
\text { grain has a grazing value of } 223 \text { sheep grazing days or four sheep per hectare for the } 8 \text { weeks immediately following } \\
\text { harvest. Agistment value of crop residues }=\text { US } \$ \text { per head/week } \times \text { weeks grazing per year } \times \text { sheep ha }{ }^{-1}=0.7 \times 8 \times 4= \\
\text { US } \$ 22.4 \text { per year. Transformity see item } 12 \text { above. }\end{array}$ \\
\hline
\end{tabular}

12 sej, eight times larger than in the tagasaste plantation system and three times larger than in the alley cropping system. Purchased energy was dominated by tractor diesel in all the systems. The tagasaste plantation system required twice as much diesel as the other two farming systems, as trees require annual pruning for efficient fodder use by animals. However, diesel represented only $3 \%$ of the total emergy flow in the plantation and $1 \%$ in the other two systems.

Dividing the total emergy flow in each farming system by the available energy (in this study measured as heat energy) of the outputs in Joules gives the transformities for each product, providing a measure of the energy efficiency of production (see also Table 6 for comparison). These showed that grain production required $20 \%$ less emergy in the alley cropping system than in the lupin/wheat rotation system, with transformities of 92700 and $117000 \mathrm{sej} / \mathrm{J}$ respectively (Tables 1,2 and 5). The emergy for nitrogen fixation varied considerably between annual and perennial systems being $46 \times 10^{12} \mathrm{sej} / \mathrm{kg}$ in the lupin/wheat rotation, $10 \times 10^{12} \mathrm{sej} / \mathrm{kg}$ in alley cropping and $3 \times 10^{12} \mathrm{sej} / \mathrm{kg}$ in the plantation.

\subsection{Sustainability ratios}

In the long run, processes with a high percent of renewable emergy are likely to be more sustainable than those with a high proportion of non-renewable emergy. The lupin/wheat rotation system was driven by a low proportion $(15 \%)$ of renewable indigenous sources (Table 6). In contrast, the flow of renewable local emergy sources amounted to $53 \%$ of the total in the tagasaste plantation system and $30 \%$ in the alley cropping system. The environmental loading ratio (the ratio of non-renewable and purchased inputs to 
Table 3

Emergy evaluation of tagasaste plantation (all flows per hectare per year)

\begin{tabular}{|c|c|c|c|c|}
\hline Item number & 1tem description & $\begin{array}{l}\text { Annual flow } \\
\text { (raw units) }\end{array}$ & $\begin{array}{l}\text { Transformity } \\
\text { (sej/unit) }\end{array}$ & $\begin{array}{l}\text { Solar emergy } \\
\left(\mathrm{sej} \times 10^{12}\right)\end{array}$ \\
\hline \multicolumn{5}{|c|}{ Renewable energy sources $(R)$} \\
\hline 1 & Sunlight $(\mathrm{J})$ & $6.89 E+13$ & $1.0 \mathrm{E}+0$ & 68.9 \\
\hline 2 & Wind, kinetic energy $(\mathrm{J})$ & $2.72 \mathbf{E}+10$ & $1.5 \mathrm{E}+3$ & 40.7 \\
\hline 3 & Rain and groundwater, chemical energy $(\mathrm{J})$ & $3.44 \mathrm{E}+10$ & $18.2 \mathrm{E}+3$ & 621.1 \\
\hline 4 & Rain, geopotential energy $(J)$ & $9.02 \mathrm{E}-08$ & $10.5 \mathrm{E}+3$ & 9.5 \\
\hline \multicolumn{5}{|c|}{ Non-renewable sources (internal) $(N)$} \\
\hline 5 & Net topsoil loss $(\mathbf{J})$ & $1.98 \mathrm{E}+09$ & $62.5 \mathrm{E}+3$ & 123.8 \\
\hline \multicolumn{5}{|c|}{ Purchased fuels and goods $(M)$} \\
\hline 6 & Diesel, gasoline and lubricants (J) & $5.49 E+08$ & $56.1 \mathrm{E}+3$ & 30.8 \\
\hline 7 & Electricity $(J)$ & $3.60 \mathrm{E}+06$ & $173.7 \mathrm{E}+3$ & 0.6 \\
\hline 8 & Phosphate $(\mathrm{kg})$ & $1.29 \mathrm{E}+01$ & $17.0 \mathrm{E}+12$ & 219.1 \\
\hline 9 & Pesticides (kg) & $4.76 \mathrm{E}+05$ & $56.1 \mathrm{E}+3$ & 0.0 \\
\hline 10 & Seed $(J)$ & $1.76 \mathrm{E}+07$ & $34.8 \mathrm{E}+3$ & 0.6 \\
\hline 11 & Machinery and buildings $(\mathrm{kg})$ & $3.12 \mathrm{E}+00$ & $3.0 \mathrm{E}+12$ & 9.4 \\
\hline \multicolumn{5}{|c|}{ Labor and service $(S)$} \\
\hline 12 & Labor (US\$) & $2.22 \mathrm{E}+01$ & $2.4 \mathrm{E}+12$ & 53.6 \\
\hline 13 & Diesel, gasoline and lubricants (US\$) & $5.21 \mathrm{E}+00$ & $2.4 \mathrm{E}+12$ & 12.6 \\
\hline 14 & Electricity (US\$) & $5.00 \mathrm{E}-01$ & $2.4 \mathrm{E}+12$ & 1.2 \\
\hline 15 & Phosphate (US\$) & $2.10 \mathrm{E}+01$ & $2.4 \mathrm{E}+12$ & 50.6 \\
\hline 16 & Pesticides (US\$) & $9.50 E-02$ & $2.4 \mathrm{E}+12$ & 0.2 \\
\hline 17 & Seed (US\$) & $1.40 \mathrm{E}+00$ & $2.4 \mathrm{E}+12$ & 3.4 \\
\hline 18 & Machinery and buildings (US\$) & $9.32 \mathrm{E}+00$ & $2.4 \mathrm{E}+12$ & 22.5 \\
\hline \multirow[t]{2}{*}{19} & Consultants, insurance and interest (US\$) & $9.40 \mathrm{E}+00$ & $2.4 \mathrm{E}+12$ & 22.7 \\
\hline & Total service and labor (US\$) & $6.91 E+01$ & & 166.7 \\
\hline \multicolumn{5}{|l|}{ Output $(Y)$} \\
\hline 20 & Tree fodder $(\mathbf{J})$ & $9.18 \mathrm{E}+09$ & $128.2 \mathrm{E}+03^{\mathrm{a}}$ & 1176.5 \\
\hline 21 & Tree residue (wood) $(\mathbf{J})$ & $6.29 \mathrm{E}+10$ & $18.76 \mathrm{E}-+03^{b}$ & 1176.5 \\
\hline 22 & Nitrogen fixation $(\mathrm{kg})$ & $3.99 E+02$ & $2.95 \mathrm{E}+12^{\mathrm{c}}$ & 1176.5 \\
\hline \multicolumn{5}{|c|}{ Economic value of output } \\
\hline 23 & Agistment value of feed (US\$) & $2.55 \mathrm{E}+02$ & $2.4 \mathrm{E}+12$ & 612.0 \\
\hline
\end{tabular}

See Table 4 for numbers and its description.

${ }^{a}$ Solar transformity of tree fodder (sej/J).

${ }^{b}$ Solar transformity of tree residues (sej/J).

c Solar transformity of nitrogen fixation $(\mathrm{sej} / \mathrm{kg}$ ).

renewable energy, Table 6) is an attempt to express the relative stress of these production systems on their environment. Adding spaced trees to the cropping system halved the environmental loading from 5.5 to 2.3 , largely due to a reduction in the emergy flow associated with wind erosion. The environmental loading for the plantation system was one-eighth of that in the lupin wheat ( 0.7 and 5.5 respectively) due to the virtual elimination of wind erosion and a two-fold increase in the amount of renewable energy directed through evapotranspiration.

\subsection{Return on investment}

The emergy investment ratio (the ratio of purchased to 'free' environmental inputs, Table 6) was similar for the lupin/wheat and the tagasaste systems ( 0.5 and 0.6 respectively), indicating that purchased materials and services amounted to about half the value of indigenous emergy sources in both cases. The alley cropping system had an investment ratio of 0.8 indicating it was almost equally dependent on purchased and 'free' inputs, reflecting the fact that while indigenous emergy 
Table 4

Item number Item description

1 Average insolation at Moora $7.25 \times 10^{9} \mathrm{~J} / \mathrm{m}^{2}$ per year (Lefroy and Stirzaker, 1999). Energy received over land $=10000 \mathrm{~m}^{2}$ (land area) $\times 5.18 \times 10^{9} \mathrm{~J} / \mathrm{m}$ per year $) \times(1-0.05)(1-$ albedo tree plantation $)=6.89 \times 10^{13} \mathrm{~J}$ per year. Transformity $=1$ by definition (Odum, 1996).

$2 \quad$ See Tables 1 and 2 item 2.

3 Precipitation $=460 \mathrm{~mm}$ per year, total evapotranspiration by trees $=650 \mathrm{~mm}$ per year transpiration $(60 \%$ from groundwater) $+46 \mathrm{~mm}$ interception (Lefroy et al., submitted for publication). No runoff and no drainage under trees. Chemical potential energy of rain over land $=10000 \mathrm{~m}^{2}$ (land area) $\times 0.696 \mathrm{~m}$ (evapotranspiration) $\times 1000 \mathrm{~kg} / \mathrm{m}^{3}$ (density of water) $\times 4940 \mathrm{~J} / \mathrm{kg}$ (Gibbs free energy of rainwater) $=3.44 \times 10^{10} \mathrm{~J}$ per year. Transformity from Odum (1996). Average amount of rainfall that ends up in the groundwater under conventional farming with annual plants is $140 \mathrm{~mm}$ per year (Asseng et al., 1998). Assuming that groundwater has the same Gibbs free energy as rainwater and that groundwater represents a split of one form of energy flow, it will have the same transformity as rain. See Tables 1 and 2 item 4.

$5 \quad$ Average soil loss from wind erosion assumed to be $1 \mathrm{~mm} / \mathrm{ha}$ per year (being a $5 \mathrm{~mm}$ loss from a major erosion event on average every 5 years $)=14$ tha per year at a bulk density of $1.4,5 \%$ of which is organic matter (including 10-100 kg/ha nitrogen), based on research by Carter et al. (1992) and Carter (unpublished data). Assuming that a tagasaste plantation provides $100 \%$ protection against wind erosion for 9 months of the year, and that during the other 3 (in autumn when the trees have been cut and are being grazed) they reduce erosion to half, soil loss is reduced to $1.4 \mathrm{~kg} / \mathrm{m}^{2}$ per year $\times 3 / 12 \times 0.5=0.175 \mathrm{~kg} / \mathrm{m}^{2}$ per year. Energy of soil loss $=10000 \mathrm{~m}^{2}$ (farmed area) $\times 0.175 \mathrm{~kg} / \mathrm{m}^{2}$ per year (erosion rate) $\times 0.05 \%$ (organic matter) $\times 5400 \mathrm{kcal} / \mathrm{kg} \times 4186 \mathrm{~J} / \mathrm{kcal}=$ $1.98 \times 19^{9} \mathrm{~J} /$ ha per year. Transformity from Odum (1996).

6 Diesel consumption $=11 \mathrm{~h} / \mathrm{h}$ for machinery operation. Operating hours for establishing and managing tagasaste plantation $=($ seeding, $5 \mathrm{~km} / \mathrm{h} \times 2.0 \mathrm{~km}$ row $/ \mathrm{ha}) / 20$ years (life of plantation $)+(1 \times$ tree pruning each year $\times 5 \mathrm{~km} / \mathrm{h} \times 2.0 \mathrm{~km} / \mathrm{ha} \times 2$ sides per row $)=1.2 \mathrm{~min}+48.0=49.2 \mathrm{~min} / \mathrm{ha} \mathrm{per} \mathrm{year} \times 11 \mathrm{l} / \mathrm{h}=9.021 / \mathrm{ha}$ per year. Energy content $=9.02 \mathrm{t}$ ha $\times 5.05 \times 10^{7} \mathrm{~J} / 1=4.56 \times 10^{8} \mathrm{~J} / \mathrm{ha}$ per year. Gasoline annual consumption involved in running 2000 ha farm $=30001$ per year $=1.51$ ha per year. Energy content $=1.5 \mathrm{l}$ /ha per year $\times$

$5.42 \times 10^{7} \mathrm{~J} / 1=8.13 \times 10^{7} \mathrm{~J} /$ ha per year. Lubricants annual consumption $=20 \mathrm{l} / 100 \mathrm{~h}$ machinery time $\times 49.2 \mathrm{~min} / \mathrm{ha}$ per year $=0.1641 /$ ha per year. Energy content $=0.164 \mathrm{~L} /$ ha per year $\times 7.38 \times 10^{7}=1.21 \times 10^{7} \mathrm{~J} /$ ha per year. Transformity from Odum (1996).

7 Annual consumption involved in running 2000 ha farm $=2000 \mathrm{kWh}=1.0 \mathrm{kWh} /$ ha per year. Energy content $=1.0 \mathrm{kWh} /$ ha per year $\times 3.6 \times 10^{6} \mathrm{~J} / \mathrm{kWh}=3.6 \times 10^{6} \mathrm{~J} /$ ha per year. Transformity from Odum (1996) Annual application $150 \mathrm{~kg} / \mathrm{ha} \times 8.6 \% \mathrm{P}=12.9 \mathrm{~kg} /$ ha per year. Transformity from Odum (1996) service subtracted.

$9 \quad$ Herbicides nil (weed control at planting achieved mechanically); Insecticides $0.17 \mathrm{~kg} / \mathrm{ha}$ per year $x$ $5.6 \times 10^{7} \mathrm{~J} / \mathrm{kg}=9.52 \times 10^{6} \mathrm{~J} /$ ha in planting year $=4.76 \times 10^{5} \mathrm{~J} /$ ha per year. Transformity for refined oil products from Odum (1996).

10 Tagasaste seed US\$28/kg. Seeding rate $1 \mathrm{~kg} / \mathrm{ha}$ to achieve a density of $\sim 2000$ trees $/$ ha. Energy content $=$ $(3000 \mathrm{kcal} / \mathrm{kg} \times 28 \mathrm{~kg} / \mathrm{ha} \times 4186 \mathrm{~J} / \mathrm{kcal}) / 20$ years (life of plantation) $=1.10 \times 10^{9} \mathrm{~J} / \mathrm{ha}$ per year. Transformity for industrial corn production, service subtracted (Odum and Odum, 1983). Total weight of machinery used in crop production $=2 \mathrm{t}$ (tractor) $=2000 \mathrm{~kg}$ (seeding and cutting carried out by contractors). Assume $1000 \mathrm{ha}$ and a life of 7 years $=0.29 \mathrm{~kg} / \mathrm{ha}$ per year. Buildings; machinery shed $(18 \mathrm{~m} \times 9 \mathrm{~m})$, super shed $(18 \mathrm{~m} \times 9 \mathrm{~m})$, total weight $4 \mathrm{t}$ steel $/ 1000$ ha plantation $=4.00 \mathrm{~kg} / \mathrm{ha}$ per year. Assume 30 years life $=0.13 \mathrm{~kg} / \mathrm{ha}$ per year. Additional $0.045 \mathrm{~km}$ fencing $/$ ha to sub-divide previously cropped area for managed grazing of tagasaste plantation $1200 \mathrm{~kg} / \mathrm{km}=54 \mathrm{~kg} / \mathrm{ha}$. Assume 20 years life $=2.7 \mathrm{~kg} / \mathrm{ha}$ per year. Total $0.13+2.7=2.83 \mathrm{~kg} / \mathrm{ha}$ per year. Transformity for refined steel products, service subtracted (Odum and Odum, 1983). Average farm income after costs and tax in 1996/1997 assumed to be $\sim$ US $\$ 20000=$ US $\$ 10 \mathrm{ha}^{-1}$ management cost for 2000 ha farm. Contract labor for tagasaste establishment and management $=\left(\right.$ US $\left.\$ 110 \mathrm{ha}^{-1}\right) / 20$ years (seeding cost) + US $\$ 8.40 \mathrm{~h} \times 0.80 \mathrm{~h} / \mathrm{ha}$ (cutting costs) $=$ US\$ $5.50 / \mathrm{ha}+$ US $\$ 6.72 \mathrm{ha}^{-1}=$ US\$ $12.22 \mathrm{ha}^{-1}$. Total cost US $\$ 22.22$ ha. Transformity used is the emergy/money ratio for the Australian economy $1996 / 1997$ (Rydberg and Lefroy, unpublished data).

13 Fuel consumption $=9.02$ tha per year. Cost $=9.021 / \mathrm{ha} \times$ US $\$ 0.461^{-1}=$ US $\$ 4.15 \mathrm{ha}^{-1}$ per year. Gasoline annual consumption involved in running 2000 ha farm $=30001$ per year $=1.5$ tha per year. Cost $=1.51$ ha per year $\times$ US $\$$ $0.531^{-1}=$ US $\$ 0.8 \mathrm{ha}^{-1}$ per year. Lubricants consumption $=201 / 100 \mathrm{~h}$ machinery time $\times 31.5 \mathrm{~min} / \mathrm{ha}$ per year $=0.105 \mathrm{l} / \mathrm{h}$ per year. Cost $=0.105$ tha per year $\times$ US $\$ 2.50 \mathrm{I}^{-1}=\mathrm{US} \$ 0.263 \mathrm{ha}^{-1}$ per year. Transformity see item 12 above. Annual consumption involved in running $2000 \mathrm{ha} \mathrm{farm}=2000 \mathrm{kWh}=1.0 \mathrm{kWh} / \mathrm{ha}$. Cost $=1.0 \mathrm{kWh} / \mathrm{ha}$ per year $\times$ LS $\$ 0.5 \mathrm{kWh}^{-1}=$ US $\$ 0.5 \mathrm{ha}^{-1}$ per year. Transformity see item 12 above. 
Table 4 (Continued)

\begin{tabular}{|c|c|}
\hline Item number & Item description \\
\hline 15 & $\begin{array}{l}\text { Arnual application } 150 \mathrm{~kg} / \mathrm{ha} \times 8.6 \% \mathrm{P}=12.9 \mathrm{~kg} / \mathrm{ha} \text { per year. Cost US } \$ 140 \mathrm{t}^{-1}=\mathrm{US} \$ \\
0.14 \mathrm{~kg}^{-1} \times 150 \mathrm{~kg} / \mathrm{ha}=\mathrm{US} \$ 21 \mathrm{ha}^{-1} \text { per year. Transformity see item } 12 \text { above. }\end{array}$ \\
\hline 16 & $\begin{array}{l}\text { Herbicides nil; insecticides }\left(0.17 \mathrm{~kg} / \mathrm{ha} \text { per year } \times \text { US } \$ 11.20 \mathrm{~kg}^{-1}\right) / 20 \text { years (life of plantation) }=\text { US\$ } 0.095 \mathrm{ha}^{-1} \\
\text { per year. Transformity see item } 12 \text { above. }\end{array}$ \\
\hline 17 & Cost of seed $=\left(\right.$ US $\left.\$ 28 \mathrm{ha}^{-1}\right) / 20$ years $=$ US $\$ 1.40 \mathrm{ha}^{-1}$ per year. Transformity see item 12 above. \\
\hline 18 & $\begin{array}{l}\text { Total annual cost of owning and maintaining machinery used in tagasaste plantation management }=\text { annual } \\
\text { depreciation }+ \text { interest on half the amount owing on a loan if it is paid off over the life of the machinery }+ \text { interest } \\
\text { on the salvage value }+ \text { maintenance costs. Assume current value in US } \$ \text { to be US } \$ 28000 \text { (tractor). Assume this } \\
\text { has a life of } 7 \text { years, a salvage value at the end of that time of US } \$ 7780 \text {, interest rate on borrowing are } 15 \% \text {, } \\
\text { which adjusted for inflation of } 5 \text { is } 10 \% \text {. Depreciation }=(28000-7780) / 7=\text { US } \$ 2888 \text {. Interest }= \\
(28000-7780) / 2 \times 10 \%=\text { US } \$ 1110 \text {. Interest on salvage value }=\text { US } \$ 7780 \times 10 \%=\text { US } \$ 778 \text {. Assume } \\
\text { maintenance costs }=\text { US } \$ 1000 \text {. Total costs }=\text { US } \$ 2888+1110+778+1000=\text { US } \$ 5766 \text {. Assume } 1000 \text { ha } \\
\text { tagasaste plantation }=\text { US } \$ 5.77 \mathrm{ha}^{-1} \text { per year. Annual cost of buildings }=\text { replacement cost/life }=\text { US } \$ \\
40000 / 30=\text { US } \$ 1300=\text { US } \$ 1.30 \mathrm{ha}^{-1} \text { per year. Annual cost of fencing }=\text { replacement cost } / \text { life }=\text { US } \$ \\
45 / 20=\text { US } 2.25 \mathrm{ha}^{-1} \text {. Total cost US } \$ 3.55 / \mathrm{ha} \text {. Transformity see item } 12 \text { above. }\end{array}$ \\
\hline 19 & $\begin{array}{l}\text { Farm management consultant paid US } \$ 1400 \text { per year }=\text { US } \$ 1.40 \mathrm{ha}^{-1} \text { per year. Insurance. US } \$ 1000 \text { per } \\
\text { year }=\text { US } \$ 0.50 \mathrm{ha}^{-1} \text { per year. Average farm debt in the cropping zone is US } \$ 150000 \text { per year. With interest rate } \\
\text { adjusted for inflation annual cost }=\text { US } \$ 15000 \text { per year }=\text { US } \$ 7.50 \mathrm{ha}^{-1} \text { per year. Transformity see item } 12 \text { above. }\end{array}$ \\
\hline 20 & $\begin{array}{l}\text { Tagasaste biomass production }=7200 \mathrm{~kg} / \mathrm{ha} \text { per year. Edible dry matter yield }=7200 \times 0.42=3024 \mathrm{~kg} / \mathrm{ha} \text { per year. } \\
\text { (Oldham and Moore, 1988; Oldham et al., 1994). Energy value }=3024 \times 725 \mathrm{kcal} / \mathrm{kg} \text { (equivalent to good quality } \\
\text { hay, Ulgiati et al. (1994)) } \times 4186 \mathrm{~J} / \mathrm{kg}=9.18 \times 10^{9} \mathrm{~J} / \mathrm{ha} \text { per year. }\end{array}$ \\
\hline 21 & $\begin{array}{l}\text { Energy value of tree residues (wood) }=7200 \mathrm{~kg} / \mathrm{ha} \times 0.58 \text { (proportion wood) } \times \\
3600 \mathrm{kcal} / \mathrm{kg} \times 4186 \mathrm{~J} / \mathrm{kg}=6.29 \times 10^{10} \mathrm{~J} / \mathrm{ha} \text { per year. }\end{array}$ \\
\hline 22 & $\begin{array}{l}\text { Fixed nitrogen measured at } 5.5 \% \text { of biomass production in plantation (Unkovich et al., submitted for publication). } \\
\text { Annual biomass production }=7200 \mathrm{~kg} / \mathrm{ha} \text {. Fixed } \mathrm{N}=7200 \times 0.055=399 \mathrm{~kg} / \mathrm{ha} \text { per year. (NB. this does not } \\
\text { include the } 100 \mathrm{~kg} \mathrm{~N} \text { taken up from the water table.) }\end{array}$ \\
\hline 23. & $\begin{array}{l}\text { Stocking rate of seven sheep per hectare based on research showing that } 1 \mathrm{~kg} \text { of tagasaste edible dry matter can } \\
\text { support one sheep for } 1 \text { day (Oldham and Moore, 1988). Edible dry matter production of } 3024 \mathrm{~kg} \text { per year is } \\
\text { therefore sufficient to support a maximum of } 3024 \text { sheep grazing days or } 8.3 \text { sheep/ha for } 1 \text { year. The inore } \\
\text { conservative rate of seven sheep per hectare has been used to allow for climate variability (drought) and feed } \\
\text { wastage. Agistment value of tree fodder = US } \$ 0.70 \text { per head per week } \times 52 \text { weeks grazing per year } \times \text { seven sheep } \\
\text { per hectare }=\text { US } \$ 254.8 \text { per year. Transformity see item } 12 \text { above. }\end{array}$ \\
\hline
\end{tabular}

Table 5

Emergy evaluation of alley cropping (all flows per hectare per year)

\begin{tabular}{|c|c|c|c|c|c|}
\hline \multirow[t]{2}{*}{ Item number } & \multirow[t]{2}{*}{ Item description } & \multicolumn{2}{|c|}{ Annual flow (raw units) } & \multirow{2}{*}{$\begin{array}{l}\text { Transformity } \\
\text { (sej/unit) }\end{array}$} & \multirow{2}{*}{$\begin{array}{l}\text { Solar emergy } \\
\left(\text { sej } \times 10^{12}\right)\end{array}$} \\
\hline & & Crop & Tree & & \\
\hline \multicolumn{6}{|c|}{ Renewable energy sources $(R)$} \\
\hline 1 & Sunlight $(\mathrm{J})$ & $4.81 \mathrm{E}+13$ & $1.03 \mathrm{E}+13$ & $1.0 \mathrm{E}+00$ & 58.4 \\
\hline 2 & Wind, kinetic energy (J) & $2.31 \mathrm{E}+10$ & $4.08 \mathrm{E}+09$ & $1.5 \mathrm{E}+03$ & 40.7 \\
\hline 3 & Rain, chemical energy $(\mathrm{J})^{\mathrm{a}}$ & $1.34 \mathrm{E}+10$ & $7.56 \mathrm{E}+09$ & $18.2 E+03$ & 381.5 \\
\hline 4 & Rain, geopotential energy $(\mathrm{J})$ & $7.67 \mathrm{E}+08$ & $1.38 \mathrm{E}+08$ & $10.5 \mathrm{E}+03$ & 9.5 \\
\hline \multicolumn{6}{|c|}{ Non-renewable sources (internal) $(N)$} \\
\hline 5 & Net topsoil loss $(\mathrm{J})^{\mathrm{b}}$ & & $5.20 \mathrm{E}+09$ & $62.5 \mathrm{E}+03$ & 324.9 \\
\hline \multicolumn{6}{|c|}{ Purchased fuels and goods $(M)$} \\
\hline 6 & Diesel, gasoline and lubricants ( $\mathrm{J}$ ) & $2.59 \mathrm{E}+08$ & $8.24 \mathrm{E}+07$ & $56.1 \mathrm{E}+03$ & 19.2 \\
\hline 7 & Electricity (J) & $3.06 \mathrm{E}+06$ & $5.40 \mathrm{E}+05$ & $174.0 \mathrm{E}+03$ & 0.6 \\
\hline 8 & Phosphate (kg) & $1.10 \mathrm{E}+01$ & $1.94 \mathrm{E}+00$ & $17.0 \mathrm{E}+12$ & 219.1 \\
\hline 9 & Pesticides (kg) & $2.31 \mathrm{E}+08$ & $7.14 \mathrm{E}+04$ & $56.1 \mathbf{E}+03$ & 13.0 \\
\hline 10 & Seed $(J)$ & $9.35 E+08$ & $2.64 \mathrm{E}+06$ & $34.8 \mathrm{E}+03$ & 32.6 \\
\hline 11 & Machinery and buildings $(\mathrm{kg})^{c}$ & $9.95 \mathrm{E}-01$ & $1.95 E-02$ & $3.0 \mathrm{E}+12$ & 3.2 \\
\hline
\end{tabular}


Table 5 (Continued)

\begin{tabular}{|c|c|c|c|c|c|}
\hline \multirow[t]{2}{*}{ Item number } & \multirow[t]{2}{*}{ Item description } & \multicolumn{2}{|c|}{ Annual flow (raw units) } & \multirow{2}{*}{$\begin{array}{l}\text { Transformity } \\
\text { (sej/unit) }\end{array}$} & \multirow{2}{*}{$\begin{array}{l}\text { Solar emergy } \\
\left(\text { sej } \times 10^{12}\right)\end{array}$} \\
\hline & & Crop & Tree & & \\
\hline \multicolumn{6}{|c|}{ Labor and service $(S)$} \\
\hline 12 & Labor (US\$) $)^{\mathrm{d}}$ & $1.14 \mathrm{E}+01$ & $2.44 \mathrm{E}+00$ & $2.4 \mathrm{E}+12$ & 33.3 \\
\hline 13 & Diesel, gasoline and lubricants (US\$) & $2.53 E+00$ & $7.82 \mathrm{E}-01$ & $2.4 \mathrm{E}+12$ & 8.6 \\
\hline 14 & Electricity (US\$) & $4.25 \mathrm{E}-01$ & $7.50 \mathrm{E}-02$ & $2.4 \mathrm{E}+12$ & 1.2 \\
\hline 15 & Phosphate (US\$) & $1.79 \mathrm{E}+01$ & $3.15 \mathrm{E}+00$ & $2.4 \mathrm{E}+12$ & 50.6 \\
\hline 16 & Pesticides (US\$) & $1.35 \mathrm{E}+01$ & $1.43 \mathrm{E}-02$ & $2.4 \mathrm{E}+12$ & 32.6 \\
\hline 17 & Seed (US\$) & $2.52 \mathrm{E}+01$ & $2.10 \mathrm{E}-01$ & $2.4 \mathrm{E}+12$ & 61.4 \\
\hline 18 & Machinery and buildings (US\$) & $2.37 \mathrm{E}+01$ & $2.00 \mathrm{E}--01$ & $2.4 \mathrm{E}+12$ & 59.7 \\
\hline \multirow[t]{2}{*}{19} & Consultants, insurance and interest (US\$) & $7.99 \mathrm{E}+00$ & $1.41 \mathrm{E}+00$ & $2.4 \mathrm{E}+12$ & 22.7 \\
\hline & Total service and labor & $1.03 \mathrm{E}+02$ & $8.28 \mathrm{E}+00$ & & 270.1 \\
\hline \multicolumn{6}{|l|}{ Output $(Y)$} \\
\hline 20 & Grain $(J)^{f}$ & $1.36 \mathrm{E}+10$ & & $92.7 \mathrm{E}+03^{\mathrm{g}}$ & 1261.1 \\
\hline 21 & Crop residues $(\mathbf{J})$ & $1.10 \mathrm{E}+10$ & & $115.0 \mathrm{E}+03^{\mathrm{h}}$ & 1261.1 \\
\hline 22 & Tree fodder $(\mathrm{J})$ & & $1.38 \mathrm{E}+09$ & $914.0 \mathrm{E}+03^{\mathrm{i}}$ & 1261.1 \\
\hline 23 & Tree residues $(\mathrm{J})$ & & $9.44 \mathrm{E}+09$ & $134.0 \mathrm{E}+03^{\mathrm{i}}$ & 1261.1 \\
\hline 24 & Nitrogen fixation $(\mathrm{kg})^{\mathrm{k}}$ & $3.40 \mathrm{E}+01$ & $9.00 \mathrm{E}+01$ & $10.2 \mathrm{E}+12^{1}$ & 1261.1 \\
\hline \multicolumn{6}{|c|}{ Economic value of output } \\
\hline 25 & Grain (US\$) & $1.25 \mathrm{E}+02$ & & $2.4 \mathrm{E}+12$ & 300.0 \\
\hline 26 & Crop residues (US\$) & $1.90 \mathrm{E}+01$ & & $2.4 \mathrm{E}+12$ & 45.7 \\
\hline \multirow[t]{2}{*}{27} & Tree fodder (US\$) & $3.83 \mathrm{E}+01$ & & $2.4 \mathrm{E}+12$ & 91.8 \\
\hline & Total value (US\$) & $1.82 \mathrm{E}+02$ & & & \\
\hline
\end{tabular}

Calculations assume $85 \%$ of inputs and outputs for crop (Tables 1 and 2) plus $15 \%$ of inputs and outputs for tagasaste plantation (Tables 3 and 4) based on the proportion of land occupied by tree and crop, except where indicated otherwise. This represents an alley cropping layout with single rows of trees $30 \mathrm{~m}$ apart and assumes no net positive or negative interactions between tree and crop when they are pruned annually for fodder utilization (Lefroy and Stirzaker, 1999). Transformities are the same as Tables 1 and 3.

${ }^{\text {a }}$ Precipitation $=460 \mathrm{~mm}$ per year, evapotranspiration by alley trees in the first year after cutting $=130 \mathrm{~mm}$ per year tree transpiration ( $60 \%$ from goundwater) $+23 \mathrm{~mm}$ tree interception; crop evapotranspiration $=272 \mathrm{~mm}$ ( $85 \%$ of sole crop) (Lefroy et al., submitted for publication). Chemical potential energy of rain over land $=10000 \mathrm{~m}^{2}$ (land area) $\times 0.153 \mathrm{~m}$ (evapotranspiration) $\times 1000 \mathrm{~kg} / \mathrm{m}^{3}(\mathrm{density}$ of water) $\times 4940 \mathrm{~J} / \mathrm{kg}$ (Gibbs free energy of rainwater) $=7.56 \times 10^{9} \mathrm{~J}$ per year (tree); $1.34 \times 10^{10} \mathrm{~J}$ per year (crop). Transformity from Odum (1996). Assuming that groundwater has the same Gibbs free energy as rainwater and that groundwater represents a split of one form of energy flow, it will have the same transformity as rain.

$\mathrm{b}$ Assumes alley cropping provides $80 \%$ protection against wind erosion for 9 months of the year and $30 \%$ during the autumn after the trees have been cut and are being grazed, based on wind speed reductions reported by Bird (1992). Average soil loss from wind erosion compared to lupin/wheat (item 5 Tables 1 and 2) is $\left(1400 \mathrm{~g} / \mathrm{m}^{2}\right.$ per year $\left.\times 9 / 12 \times 0.2\right)+\left(1400 \mathrm{~g} / \mathrm{m}^{2}\right.$ per year $\left.\times 3 / 12 \times 0.7\right)=$ $0.21+0.25=460 \mathrm{~g} / \mathrm{m}^{2}$ per year. Energy of soil loss $=10000 \mathrm{~m}^{2}$ (farmed area) $\times 0.46 \mathrm{~kg} / \mathrm{m}^{2}$ per year (erosion rate) $\times 0.05 \%$ (organic matter) $\times 5400 \mathrm{kcal} / \mathrm{kg} \times 4186 \mathrm{~J} / \mathrm{kcal}=5.20 \times 10^{9} \mathrm{~J} / \mathrm{ha}$ per year.

c Crop: $85 \%$ of conventional cropping. Tree: $15 \%$ of buildings used in plantation calculation less extra fencing which is only required for intensive grazing of plantation tagasaste $=0.13 \mathrm{~kg} / \mathrm{ha} \times 0.15=0.0195 \mathrm{~kg} / \mathrm{ha}$.

${ }^{d}$ Crop: $85 \%$ of conventional cropping. Tree: Contract labor for tagasaste establishment and management $=($ US $\$ 110 / \mathrm{ha} \times 0.2) / 20$ years (seeding cost) + US $\$ 8.40 \mathrm{~h}^{-1} \times 0.16 \mathrm{~h} / \mathrm{ha}$ (cutting costs) $=$ USS $1.10 \mathrm{ha}^{-1}+\mathrm{US} \$ 1.34 \mathrm{ha}^{-1}=\mathrm{US} \$ 2.44 \mathrm{ha}^{-1}$. Average farm income after costs and tax in $1996 / 1997 \sim$ US $\$ 20000=$ US $\$ 10 \mathrm{ha}^{-1}$ cost of managing a 2000 ha farm. Total cost $=$ labor for cropped area + US $\$$ $2.44 \mathrm{ha}^{-1}$.

${ }^{\mathrm{e}}$ Crop: $85 \%$ of conventional cropping. Tree: $15 \%$ of buildings used in plantation calculation less extra fencing which is only required for intensive grazing of plantation tagasaste $=$ US $\$ 1.30 \mathrm{ha}^{-1} \times 0.15=$ US\$ $0.20 \mathrm{ha}^{-1}$ per year.

$f$ Lupin yield is $85 \%$ of sole crop district average $(680 \mathrm{~kg} / \mathrm{ha})$. Cereal yield is $85 \%$ of district average $(1275 \mathrm{~kg} / \mathrm{ha})$. Total over $2 \mathrm{years}$ of rotation is 1955 , annual equivalent $=978 \mathrm{~kg} /$ ha per year. Energy content $=978 \times 1.39 \times 10^{7} \mathrm{~J} / \mathrm{kg}=1.36 \times 10^{10} \mathrm{~J} / \mathrm{ha}$.

g Solar transformity of grain (sej/J).

h Solar transformity of crop residue (sej/J).

i Solar transformity of tree fodder $(\mathrm{sej} / \mathrm{J})$.

j Solar transformity of tree residues (sej/J).

${ }^{k} \mathrm{~N}$ fixation by trees in alley crop measured at $90 \mathrm{~kg} \mathrm{~N} / \mathrm{ha}$ per year (total biomass plus leaf litter, Unkovich et al., submitted for publication). Assume fixation by crops is $85 \%$ of sole crop $=40 \times 0.85 \mathrm{~kg} / \mathrm{ha}$ per year $=34$. Total $=90+34=124 \mathrm{~kg} / \mathrm{ha}$. per year.

Solar transformity of nitrogen fixation $(\mathrm{sej} / \mathrm{kg})$. 
Table 6

Transformities emergy indices and money flows for the three alternative farming systems

\begin{tabular}{|c|c|c|c|c|}
\hline Index & Expression & $\begin{array}{l}\text { Lupin/wheat } \\
\text { rotation }\end{array}$ & $\begin{array}{l}\text { Tagasaste } \\
\text { plantation }\end{array}$ & $\begin{array}{l}\text { Alley } \\
\text { cropping }\end{array}$ \\
\hline \multicolumn{5}{|l|}{ Transformities } \\
\hline Grain (sej/J) & & $117.0 \mathrm{E}+3$ & & $92.7 \mathrm{E}+3$ \\
\hline Crop residues (sej/J) & & $144.2 \mathrm{E}+3$ & & $115.0 \mathrm{E}+3$ \\
\hline Tree fodder $(\mathrm{sej} / \mathrm{J})$ & & & $128.2 \mathrm{E}+3$ & $914.0 \mathrm{E}+3$ \\
\hline Tree residues $(\mathrm{sej} / \mathrm{J})$ & & & $18.8 \mathrm{E}+3$ & $134.0 \mathrm{E}+3$ \\
\hline Nitrogen fixation $(\mathrm{sej} / \mathrm{kg}$ ) & & $46.5 \mathrm{E}+12$ & $3.0 \mathrm{E}+12$ & $10.2 \mathrm{E}+12$ \\
\hline \multicolumn{5}{|l|}{ Sustainability ratios } \\
\hline Renewable proportion of total emergy & $R / Y$ & 0.2 & 0.6 & 0.3 \\
\hline Environmental loading (ELR) & $(N+M+S) / R$ & 5.5 & 0.7 & 2.3 \\
\hline \multicolumn{5}{|l|}{ Emergy investment ratios } \\
\hline Investment ratio & $(M+S) /(R+N)$ & 0.5 & 0.6 & 0.8 \\
\hline Emergy exchange ratio & $Y /$ output price in emergy & 4.5 & 1.9 & 2.9 \\
\hline Return on invested emergy & Output price in emergy/ $(M+S)$ & 0.7 & 1.0 & 0.7 \\
\hline \multicolumn{5}{|l|}{ Gross margin (US\$) } \\
\hline Value of output (US\$ ha-1 per year) & & 170 & 255 & 182 \\
\hline Purchased inputs and services (US\$ ha- $\mathrm{h}^{-1}$ per year) & & 121 & 69 & 103 \\
\hline Net income (US\$ ha ${ }^{-1}$ per year) & & 49 & 186 & 79 \\
\hline
\end{tabular}

To avoid double counting, the aggregated flow of renewable indigenous resources $(R)$ is taken to be the largest renewable flow (items $1-4$, Tables 1-5), as the other flows represent by-products of the same coupled processes.

sources were reduced compared to the lupin/wheat system through lower wind erosion, purchased inputs remained similar.

The emergy exchange ratio (the solar emergy value of the total yield divided by the solar emergy value of the currency, Table 6) provides a measure of the emergy content per dollar of final product. This was found to be 4.5 for the lupin/wheat system, 1.9 for the plantation and 2.9 for alley crop systems respectively. This shows that the prices received for the products of all farming systems under value their environmental inputs. Virtually, all the products that have been studied using this method have a higher emergy content than is reflected in their dollar value (Odum, 1996). The main reason for the high exchange ratio in the lupin/wheat rotation in this study was the soil loss. As this is not taken into account in a purely economic analysis, it is not reflected in the purchase price of the grain.

The return on invested emergy (the ratio of the amount of emergy that can be purchased with the income received for the products to the emergy content of purchased goods and services in the economy, Table 6) was 0.7 in the lupin/wheat and alley cropping systems and 1.0 in the tagasaste plantation system.
This reflects the terms of trade between the farming system and the broader economy in energy units. A ratio above 1 indicates that the system gains in trade, in that the farmer receives more emergy in the form of payments for the product that is needed to run the system. A ratio smaller than 1 indicates that less emergy is paid for the products than is needed run the system. In this sense, the terms of trade were neutral in the tagasaste plantation and negative in the other two systems.

The gross margin analysis indicated that the tagasaste plantation had an annual net return of almost four times that of the lupin/wheat system, while net returns from alley cropping were $45 \%$ higher than from the lupin/wheat system (Table 6).

\section{Discussion}

The aim of this study was to use Odum's (1996) emergy method to compare the resource use and environmental impact of three alternative farming systems as measures of their relative sustainability. This method was selected as it potentially provides a more comprehensive method of assessment than is possible 
when tracking material cycles such as (e.g. water and nutrients) or money flows. The results provide as much insight into the assumptions inherent in this approach as they do into the farming systems under study.

Wind erosion emerged as the primary reason for the differences in energy flow and sustainability between the lupin/wheat, plantation and alley cropping systems. In the plantation and alley cropping systems, trees reduced the non-renewable energy flows and the environmental loading to one-eighth and one-third, respectively of the values in the lupin/wheat system. An argument against the assumption that net soil loss occurs through wind erosion is that the eroded soil would be deposited on each hectare at the same rate as it was removed. However, the nature of wind erosion events in this environment is that the organic matter fraction upon which the emergy analysis is based accumulates along fence lines, vegetation strips and any other slight impediments to wind flow. It is therefore effectively lost to the cropping system and represents a net outflow of energy.

The total energy flows associated with evapotranspiration were far lower than those associated with wind erosion. More significantly there is less potential to improve the ratio of renewable to non-renewable energy use, and therefore long term sustainability in energy terms, by increasing tree water use. Even if tree water use was assumed to be $1200 \mathrm{~mm}$ per year, at the upper limit for this environment (Raper, 1998), the maximum impact on energy flows would be half that of reducing wind erosion. This would also require leaving the trees un-cut and forfeiting the economic value of tree fodder.

The relative importance placed on wind erosion over water use in this analysis contrasts with the perceived impact of these two processes in the real world. Wind erosion is considered to produce a temporary reduction in productivity (Carter et al., 1992) while the impact of rising water tables is effectively permanent. From the perspective of emergy analysis, a smaller total energy flow is involved in returning water to the atmosphere than in producing soil organic matter. This reflects the scale at which this study was carried out rather than challenging us to reconsider the relative importance we place on these two processes. To fully incorporate the effects of rising water tables on productivity, analysis would need to be carried out at catchment scale or larger, including the rate of conversion of arable land to waterlogged ecosystems. This would require specifying the proportion of the landscape under various vegetation types (annual, perennial, waterlogged), the water use of each vegetation type, and also involve assumptions about the discharge capacity of the catchment and the rate of sub-surface water movement, processes that are poorly understood.

The higher value placed on soil loss over water management also reflects the supply driven nature of the emergy method, whereby processes are valued according to the number and kind of energy transformations involved, rather than their consequences or utility as seen from a human perspective. This follows from the maximum power principle based on the work of Lotka (1925) that lies at the heart of emergy analysis. This principle holds that systems that prevail in competition with others are those that can obtain and use energy most effectively (Odum, 1994, 1988).

One of the practical consequences of the supply driven and hierarchical structure of emergy analysis is the difficulty of calculating transformities, the cumulative amount of energy of all forms used to generate each Joule or kilogram of an input or output. Smil (1991) and Cleveland (1992) highlight the problem of defining the spatial and temporal boundaries necessary to calculate the total energy required to produce inputs such as soil, phosphate and fossil fuels. In the case of fossil fuels, this potentially includes the photosynthetic energy that generated the biomass, the radiation that energized erosion and sedimentation, and the radioactive decay that powers tectonic movement. Despite the lack of agreement over its basic assumptions and the difficulties in calculating transformities, Brown and Herendeen (1996) concluded in a comparative review that emergy analysis is a bolder and more comprehensive synthesis of the interdependencies driving ecological and economic systems than conventional embodied energy analysis.

Where the same method is used to derive transformities, they can be used to compare the energy use efficiency of production processes. In Table 7 the transformities and environmental loading for the three farming systems in this study are compared with those from reported other studies. Grain production in the lupin/wheat and alley cropping systems with transformities of 117000 and $92700 \mathrm{sej} / \mathrm{J}$ respectively was more efficient than wheat produced in Italy with a transformity of $159000 \mathrm{sej} / \mathrm{J}$ (Ulgiati et al., 1994). 
Table 7

Transformity for grain and fodder and environmental loading ratio for the three cropping systems compared with those reported from the other studies

\begin{tabular}{lcll}
\hline Crop/farming system & Transformity $(\mathrm{sej} / \mathrm{J})$ & Environmental loading ratio & Reference \\
\hline Grain in lupin/wheat rotation & $11.7 \mathrm{E}+4$ & 5.5 & This study \\
Tree fodder in tagasaste plantation & $12.8 \mathrm{E}+4$ & 0.7 & This study \\
Alley cropping & & 2.3 & This study \\
Grain in alley cropping & $9.3 \mathrm{E}+4$ & & This study \\
Tree fodder in alley cropping & $91.4 \mathrm{E}+4$ & 3.4 & This study \\
Wheat, Italy & $15.9 \mathrm{E}+4$ & 1.5 & Ulgiati et al. (1994) \\
Forage, ltaly & $8.0 \mathrm{E}+4$ & 0.9 & Ulgiati et al. (1994) \\
Soybean, Italy & $4.0 \mathrm{E}+4$ & 1.0 & Panzieri et al. (2000) \\
Hay, Sweden & $1.4 \mathrm{E}+4$ & Rydberg and Jansén (2002) \\
\hline
\end{tabular}

The environmental loading however (the proportion of non-renewable and purchased inputs to 'free' inputs) was higher for the lupin/wheat rotation (5.5) than Italian wheat production (3.4) and lower for alley cropping (2.3). Ulgiati et al. (1994) reported a transformity of $80000 \mathrm{sej} / \mathrm{J}$ and an environmental loading of 1.5 for forage produced in Italy. While the transformity for forage in that study was lower than the transformity calculated for tree fodder from tagasaste plantation, the environmental loading ratio was higher than that of the tagasaste forage.

The high transformity (i.e. low energy use efficiency) of fodder production in the plantation system (128 $000 \mathrm{sej} / \mathrm{J}$ ) can be regarded as a measure of the energy investment in permanent root systems and woody stems necessary to improve the sustainability of land use in this environment. When the transformity for each farming system is expressed in terms of total above ground biomass as opposed to grain or tree fodder, the plantation was the most efficient $(16300 \mathrm{sej} / \mathrm{J})$ compared to the lupin/wheat system $(48000 \mathrm{sej} / \mathrm{J})$ and alley cropping $(47500 \mathrm{sej} / J)$. Hay production in Sweden has been reported with a similarly low transformity and a low environmental loading ratio to that of the tagasaste plantation system (Table 7).

The higher returns and more favorable sustainability ratios in the tagasaste plantation suggests a win:win situation for any landholder who makes the transition from annual cropping to tagasaste plantation. However, a feature of agroforestry systems not reflected in annual gross margins or this energy analysis is the time lag between the establishment and full production. While the amortized costs of establishing the agroforestry systems were included, the debt that would have to be carried over the 4-5 years prior to full production was not, and remains a major obstacle to adoption (Taylor et al., 1996; Lefroy and Stirzaker, 1999; Pannell, 1999).

\section{Conclusion}

Emergy analysis carried out on three farming systems at a 1 ha scale indicated that the largest energy flows in all systems were those associated with wind erosion, evapotranspiration and application of phosphate fertilizer. A plantation system based on the fodder tree tagasaste (Chamaecytisus proliferus) had the lowest environmental loading (ratio of non-renewable to renewable resource inputs) due to reduced wind erosion and the highest annual net returns compared to annual cropping. An alley cropping system with $15 \%$ tree cover was intermediate according to both sets of criteria. The environmental consequences of annual cropping systems in western Australia have occurred rapidly after their introduction, with soil erosion and rising water tables the most significant consequences. Perennial plant-based land use systems are required to address both issues by providing year round physical protection of the soil surface and increased rates of evapotranspiration. Emergy analysis provides an instrument that combines environmental and economic assessment of prospective land use systems in one analytical framework by evaluating both the environmental contributions considered free from a market perspective and those nested to monetary flows. While this potentially serves as a good base for policy decisions aimed at encouraging more sustainable land 
use systems, to fully incorporate the off-site effects of rising water tables in the agricultural landscapes studied, a larger scale analysis would be required.

\section{Acknowledgements}

This study was made possible by an OECD Cooperative Research Fellowship (EL) and financial support from the Faculty of Agriculture, Landscape Planning and Horticulture at the Swedish Agricultural University (TR). We thank Steven Doherty, University of Florida, and two anonymous reviewers.

\section{References}

Altieri, M.A. (Ed.), 1987. Agroecology-The Scientific Basis of Alternative Agriculture. Westview Press, Boulder.

Anderson, G.C., Fillery, I.R.P., Dunin, F.X., Dolling, P.J., Asseng, S., 1998. Nitrogen and water flows under pasture-wheat and lupin-wheat rotations in deep sands in western Australia. 2. Drainage and nitrate leaching. Aust. J. Agric. Res. 49, 345-362.

Asseng, S., Fillery, I.R.P., Anderson, G.C., Dolling, P.J., Dunin, F.X., Keating, B.A., 1998. Use of the APSIM wheat model to predict yield, drainage and $\mathrm{NO}_{3}$-leaching for a deep sand. Aust. J. Agric. Res. 49, 363-377.

Australian Bureau of Agricultural and Resource Economics, 1997. Australian Commodity Statistics. ABARE, Canberra.

Australian Bureau of Meteorology, 1977. Manual of Meteorology. Department of Science and Technology, Australian Government Publishing Service, Canberra, $149 \mathrm{pp}$.

Bird, P.R., 1992. The role of shelter in Australia for protecting soils, plants and livestock. Agrofor. Syst. 20, 59-86.

Brown, M.T., Herendeen, J., 1996. Embodied energy analysis and emergy analysis: a comparative view. Ecol. Econ. 19, 219-235.

Brown, M.T., Ulgiati, S., 1998. Emergy Evaluation of the Environment: quantitative perspectives on ecological footprints. In: Ulgiati, S., Brown, M.T. Giampietro, M., Herendeen, R.A., Mayumi, K. (Eds.). Advances in Energy Studies. Energy Flows in Ecology and Economy. MUSIS, Roma, pp. 223-240.

Brown, M.T., Ulgiati, S., 2001. Emergy measures of carrying capacity to evaluate economic investments. Populat. Environ. 22 (5), 471-501.

Brown, M.T., Brandt-Wiliams, S., Tilley, D., Ulgiati, S. (Eds.), 2000. Energy synthesis. Theory and applications of the emergy methodology. In: Proceedings of the First Biennial Emergy Analysis Research Conference, Gainesville, FL, September 1999, 328 pp.

Carter, D., Findlater, P., Porritt, S., 1992. Stubble retention for control of wind erosion. J. Agric. (WA) 33 (1), 15-17.

Cleveland, C.J., 1992. Energy quality and energy surplus in the extraction of fossil fuels in the US. Ecol. Econ. 6, 139-162.
Conway, G.C., 1985. Agricultural ecology and farming systems research. In: Remenyi, J.V. (Ed.), Proceedings of the ACIAR, Agricultural Systems Research for Developing Countries, no. 11.

Costanza, R., d'Arge, R., deGroot, R., Farber, S., Grasso, M., Hannon, B., Limburg, K., Naeem, S., O'Neill, R., Paruelo, J., Raskin, R.G., Sutton, P., van den Belt, M., 1997. The value of the world's ecosystem services and natural capital. Nature $387,253-260$.

Edwards-Jones, G., Davis, B., Hussain, S., 2000. Ecological Economics an Introduction. Blackwell Scientific Publications, Oxford.

Fluck, R.C., Baird, D.C., 1980. Agricultural Energetics. Avi Publishing Company, Westport, CT, 192 pp.

George, R.J., McFarlane, D.J., Nulsen, R.A., 1997. Salinity threatens the viability of agriculture and ecosystems in Western Australia. Hydrogeol. J. 5, 6-21.

Hatton, T.J., Nulsen, R.A., 1999. Towards achieving functional ecosystem minicry with respect to water cycling in southern Australian agriculture. Agrofor. Syst. 45, 203-214.

Lefroy, E.C., 1994. A tale of two alleys: parallel evolution in agroforestry systems. Agrofor. Today 6, 5-7.

Lefroy, E.C., Stirzaker, R.J., 1999. Agroforestry for water management in southern Australia. Agrofor. Syst. 45, 277-302.

Lefroy, E.C., Hobbs, R.J., O'Connor, M.H., Pate, J.S., 2000. Agriculture as a Mimic of Natural Ecosystems. Kluwer Academic Publishers, Dordrecht. The Netherlands, 492 pp.

Lefroy, E.C., Pate, J.S., Stirzaker, R.J., 2001 a. Growth, water use efficiency and adaptive features of tagasaste (Chamaecytisus proliferus) at alley and plantation densities. Aust. J. Agric. Res. 52, 221-234.

Lefroy, E.C., Stirzaker, R.J., Pate, J.S., 2001b. The influence of tagasaste (Chamaecytisus proliferus) trees on the water balance of an alley cropping system. Aust. J. Agric. Res. 52, 235-246.

Lotka, A.J., 1925. Physical Biology. Williams and Wilkins, Baltimore.

Odum, H.T., 1988. Self-organization, transformity and information. Science 242, 1132-1139.

Odum, H.T., 1994. Ecological and General Systems: An Introduction to Systems Ecology. University Press of Colorado, revised edition of Systems Ecology, 1983, Wiley, New York, $644 \mathrm{pp}$.

Odum, H.T., 1996. Environmental Accounting: Emergy and Environmental Decision Making. Wiley, New York, 370 pp.

Odum, H.T., 1998. Emergy evaluation. In: Ulgiati, S., Brown, M.T., Giampietro, M., Herendeen, R.A., Mayumi, K. (Eds.), Advances in Energy Studies. Energy Flows in Ecology and Economy. MUSIS, Roma. pp. 99-111.

Odum, H.T., Odum, E.C., 1983. Energy Analysis Overview of Nations: Concepts and Methods. Working Paper. International Institute of Applied Systems Analysis, Laxenburg, Austria, 469 pp.

Odum, H.T., Odum, E.C., 2001. A Prosperous Way Down. Principles and Policies. University Press of Colorado, Boulder, $326 \mathrm{pp}$.

Odum, H.T., Brown, M.T., Ulgiati, S., 2000. Ecosystems as energetic systems. In: Jørgensen, S.E., Müller, F. (Eds.), Handbook of Ecosystem Theories and Management. Lewis Publisher, Boca Raton, pp. 281-302. 
Oldham, C.M., Moore, P.M., 1988. Tagasaste (Chamaecytisus palmensis) an evergreen fodder tree in grazing systems of Mediterranean type climates. 2. The feeding value for wool production when grazed by young merino ewes over summer and autumn. Proc. Int. Grassl. Congr. XVI, 1251-1252.

Oldham, C.M., Allen, G., Fortune, J.A., 1994. Production from cows and calves set stocked on tagasaste, a perennial leguminous fodder shrub. Proc. Aust. Soc. Anim. Prod. 20, $85-88$.

Pannell, D., 1999. Social and economic constraints to the adoption of complex farming systems. Agrofor. Syst. 46, 393-409.

Panzieri, M., Marchettini, N., Hallam, T.G., 2000. Importance of the Bradhyrizobium japonicum symbiosis for the sustainability of a soybean cultivation. Ecol. Model. I35, 301-310.

Pearce, D.W., 1983. Cost-benefit Analysis. Macmillan, Bath.

Pearce D.W., Turner, R.K., 1990. Economics of Natural Resources and the Environment. Harvester Wheatsheaf, Hemel Hempstead.

Pimentel, D.C., Wilson, C., McCullum, R., Huang, P., Dwen, J., Flack, Q., Tran, T., Saltman, T., Cliff, B., 1997. Economic and environmental benefits of biodiversity. Bioscience 47, 747-758.

Raper, P., 1998. Agroforestry Water Use in Mediterranean Regions of Australia. Rural Industries Research and Development Corporation, Publication 98/063, 71 pp.
Smil, V., 1991. General Energetics: Energy in the Biosphere and Civilization. Wiley, New York.

Rydberg, T., Jansén, J., 2002. Comparison of horse and tractor traction using emergy analysis. Ecol. Eng. 19, 13-28.

Stout, B.A., 1990. Handbook of Energy for World Agriculture. Elsevier, London, 504 pp.

Taylor, E., Cook, J., Dunham, C., Leeson, R., Park, D., Wilson, R., 1996. Management and investment strategies for tagasaste: the farmers' perspective. In: Lefroy, E.C., Oldham, C.M., Costa, N.J. (Eds.). Tagasaste (Chamaecytisus proliferus). Centre for Legumes in Mediterranean Agriculture, Perth, pp. 211-220.

Ulgiati, S., Odum, H.T., Bastianoni, S., 1994. EMERGY use, environmental loading and sustainability. An EMERGY Analysis of Italy. Ecol. Model. 73, 215-268.

Unkovich, M.J., Pate, J.S., Lefroy, E.C., Arthur, D.J., 2000. Inputs of fixed $\mathrm{N}$ by the fodder tree legume tagasaste (Chamaecytisus proliferus) in deep sands of Western Australia assessed using the I5N natural abundance technique. Aust. J. Plant. Physiol. $27,921-929$.

Williams, J., I997. Re-inventing Australia's Plant Production Systems. CSIRO Land and Water, Canberra. 